\title{
Humidity Sensing Properties of Surface Modified Polyaniline Metal Oxide Composites
}

\author{
S. C. Nagaraju, ${ }^{1,2}$ Aashis S. Roy, ${ }^{3}$ J. B. Prasanna Kumar, ${ }^{4}$ \\ Koppalkar R. Anilkumar, ${ }^{5}$ and G. Ramagopal ${ }^{6}$ \\ ${ }^{1}$ Department of Physics, Bharathiar University, Coimbatore, Tamil Nadu 641046, India \\ ${ }^{2}$ Department of Physics, Vidyavahini College, SIT Extension, Tumkur, Karnataka 572102, India \\ ${ }^{3}$ Department of Materials Science, Gulbarga University, Gulbarga, Karnataka 585106, India \\ ${ }^{4}$ Department of Physics, GFGC Tumkur, Tumkur, Karnataka 572102, India \\ ${ }^{5}$ Department of Physics, GFG Degree College, Shahabad, Gulbarga, Karnataka 585228, India \\ ${ }^{6}$ Department of Physics, Maharani’s Science College, Palace Road, Bangalore, Karnataka 560 001, India
}

Correspondence should be addressed to G. Ramagopal; dr_gramgopal@yahoo.co.in

Received 7 May 2014; Revised 16 August 2014; Accepted 30 September 2014; Published 25 November 2014

Academic Editor: Sadhan C. Jana

Copyright (C) 2014 S. C. Nagaraju et al. This is an open access article distributed under the Creative Commons Attribution License, which permits unrestricted use, distribution, and reproduction in any medium, provided the original work is properly cited.

Polyaniline- (PANI) praseodymium Oxide $\left(\mathrm{Pr}_{2} \mathrm{O}_{3}\right)$ composites have been synthesized by in situ polymerization method with different weight percentages. The synthesized composites have been characterized by Fourier transform infrared spectroscopy, Xray diffraction and scanning electron microscopy. The temperature dependent conductivity shows that the conductivity is due to the hopping of polarons and bipolarons. These composites show negative thermal coefficient $(\alpha)$ behavior as a function of temperature, which is characteristic behavior of semiconducting materials. Sensor studies have been carried out by two-probe method and found that the sensitivity increases with increase in \% RH. It is noticed that stability increase is due to the presence of $\operatorname{Pr}_{2} \mathrm{O}_{3}$ in polyaniline up to $30 \mathrm{wt} \%$. A fast recovery and response time along with high sensitivity make these composites suitable for humidity sensors.

\section{Introduction}

Conducting polymer has achieved more attention towards the humidity sensing application in present era due to its low weight, easy processing, and high absorption capability because of its porous nature [1]. Among the large variety of conducting polymers, polyaniline (PANI) has emerged as the most promising one because of its diverse properties like relatively inexpensive monomer, easy preparation, high yield of polymerization, environmental stability, and unique processability for device fabrication $[2,3]$. The various techniques such as electrochemical polymerization, chemical oxidation, and electrochemical deposition and spin coating have been explored for the fabrication of polymer sensors [4]. Advantages with polymers as sensing materials are light weight, flexible, low cost, and simple fabrication process. Polyaniline has shown very promising results for sensing applications in comparison to other classical sensor materials employed in the fabrication of sensors $[5,6]$. Various oxide doped polymer composite materials have been examined as the humidity sensor; for example, $\mathrm{ZnO}, \mathrm{CdO}$, and $\mathrm{WO}_{3}$ are reported to exhibit high sensitivity toward humidity $[7,8]$. However, further improvements in the performance of the mixed-potential type polymer sensors are still needed in order to realize a commercially viable device of this type [9]. Basically, polymer metal oxide is used in humidity sensor applications and is prepared by conventional and advanced wet chemical processing methods at room temperature and is mainly developed to offer porous bodies. The advantage of an absorbent spongiform surface rather than a condensate is a greater permeability of water molecules, so water vapor molecules can easily pass through the pore openings and capillary condensation occurs in the capillary porous structures which are formed between the grain distributions in ceramic surface during the pore removal process. 
The proper control of the surface composition of polyaniline-metal oxide humidity sensors is essential for obtaining rapid, reliable, and selective response to the adsorption of water vapor [10]. As a result of the compositional changes, the nature and extent of acidic and basic sites on the surface are altered drastically and often the resultant oxide surface behaves as a better proton acceptor/donor depending on the nature of the dopants, leading to enhanced humidity sensitivity [11-13]. Parvatikar et al. synthesized composites of PANI with metal oxides such as $\mathrm{WO}_{3}[14], \mathrm{Co}_{3} \mathrm{O}_{4}$ [15], and $\mathrm{CeO}_{2}[16]$ by using chemical polymerization and investigated the humidity sensing properties. The electrical and optical properties of polyaniline are easily tunable by doping inorganic or organic compound [17-19]. These properties show a possibility of using these polymers as a humidity sensing materials. Various researches have shown that conducting polymer-metal oxide nanocomposites are also convincing humidity sensors because of their good stability and high surface to volume ratio [18-20].

In this work, $\mathrm{PANI}-\mathrm{Pr}_{2} \mathrm{O}_{3}$ composites have been prepared by in situ polymerization method with various weight percentages. The structural property and surface morphology have been analyzed by using Fourier transform infrared spectroscopy (FTIR), X-ray diffraction (XRD), and scanning electron microscope (SEM). Further the DC conductivity and humidity sensing behavior of these composites have been studied by using Kelvin two-probe techniques.

\section{Experimental}

All chemicals used for synthesis are of analytical reagent (AR) grade. Aniline, ammonium peroxydisulphate $\left(\mathrm{NH}_{4}\right)_{2} \mathrm{~S}_{2} \mathrm{O}_{8}$; (APS), hydrochloric acid ( $\mathrm{HCl}$ ), and $\mathrm{Pr}_{2} \mathrm{O}_{3}$ have been purchased from Sigma-Aldrich, India. Aniline monomer is doubly distilled before use. The composite samples so synthesized are processed into circular pellets of $10 \mathrm{~mm}$ diameter and $2 \mathrm{~mm}$ thickness by applying pressure of 10 ton in a UTM40 machine (40 Ton Universal Testing Machine) using a hydraulic press. For conductivity measurements, the pellets are coated with silver paste on either side of the surfaces to provide good electrical contacts.

2.1. Synthesis of Polyaniline. Aniline $(0.1 \mathrm{~mol})$ is dissolved in $1 \mathrm{M}$ hydrochloric acid for $20 \mathrm{~min}$ to form aniline hydrochloride. The above solution is refluxed with $1 \mathrm{~mL}$ of camphor sulphonic acid (CAS) which acts as a surfactant for 3 hours at $0^{\circ} \mathrm{C}$ to $5^{\circ} \mathrm{C}$. Further, $0.1 \mathrm{M}$ of ammonium persulfate $\left[\left(\mathrm{NH}_{4}\right)_{2} \mathrm{~S}_{2} \mathrm{O}_{8}\right]$ is added to the refluxed solution which acts as the oxidant and is added drop-wise with continuous stirring for 6 hours at ice temperature and is kept 24 hours without stirring for complete polymerization. The green precipitate powder is recovered using vacuum filter and washed with $0.1 \mathrm{HCl}$ in order to remove the excess chlorine ions followed by water and acetone to remove the excess ammonium persulfate and unreacted monomers from the composites. Finally the resultant precipitate is dried under dynamic oven for 24 hours to achieve a constant weight [21, 22].
2.2. Synthesis of PANI-Pr $\mathrm{O}_{3}$ Composites. $0.1 \mathrm{~mol}$ aniline is dissolved in $1 \mathrm{M}$ hydrochloric acid and to this known amount of $\operatorname{Pr}_{2} \mathrm{O}_{3}(10,20,30,40$, and $50 \mathrm{wt} \%)$ is added and stirred for $20 \mathrm{~min}$ to form metal oxide suspended aniline hydrochloride. The above solution is refluxed for 3 hours at $0^{\circ} \mathrm{C}$ to $5^{\circ} \mathrm{C}$ with $1 \mathrm{~mL}$ of camphor sulphonic acid (CAS) which acts as a surfactant. Further, $0.1 \mathrm{M}$ of ammonium persulfate $\left[\left(\mathrm{NH}_{4}\right)_{2} \mathrm{~S}_{2} \mathrm{O}_{8}\right]$ is added to the refluxed solution which acts as an oxidant and added drop-wise with continuous stirring for 6 hours at ice temperature and is kept 24 hours without stirring to complete the polymerization. The green precipitate powder of PANI$\mathrm{Pr}_{2} \mathrm{O}_{3}$ composite is recovered by using vacuum filter and washed with $0.1 \mathrm{HCl}$ in order to remove the excess chlorine ions followed by water and acetone to remove the excess ammonium persulfate and unreacted monomers from the composites. Finally the resultant precipitate is dried under dynamic oven for 24 hours to achieve a constant weight.

\section{Characterization}

The above synthesized PANI- $\mathrm{Pr}_{2} \mathrm{O}_{3}$ composites are structurally and surface morphologically characterized by using different techniques like FTIR, X-ray diffraction (XRD), and scanning electron microscopy.

The FTIR spectra of polyaniline and its composites are recorded on Perkin Elmer (model 783) IR spectrometer in $\mathrm{KBr}$ medium at room temperature. For recording FTIR spectra, sample powders are mixed with $\mathrm{KBr}$ in the ratio of $1: 25$ by weight and grounded to ensure the uniform dispersion of samples in $\mathrm{KBr}$ pellets. The mixed powders are pressed in a cylindrical dye to obtain clean discs of approximately $1 \mathrm{~mm}$ thickness [23].

The X-ray diffraction patterns of the prepared samples are obtained by employing Philips X-ray diffractometer using $\mathrm{CuK}_{\alpha}$ radiation $(\lambda=1.5406 \AA)$. The diffractograms are recorded in terms of $2 \theta$ in the range $10^{\circ}-80^{\circ}$ with a scanning rate of $2^{\circ}$ per minute.

The surface morphology of polyaniline and its composites are studied by using Phillips XL30 ESEM scanning electron microscope (SEM). The powder samples are dispersed on the surface of carbon tape mounted on aluminum tab and conducting gold is sputtered on the sample to avoid charging at the sample surfaces and hence selected areas are photographed.

3.1. DC Conductivity Measurements. A typical experimental setup used for the measurement of dc conductivity consists of a sample holder securely locating the sample coated with silver paste in order to get better contact inserted from top aperture of the heating furnace. A potential difference is applied across the sample. The electrodes and thermocouple leads are taken out from top aperture and connected to Keithely 2100 electrometer to measure the change in current of the samples and temperature indicator, respectively. A heater placed at the bottom of the furnace is capable of giving a variable but linear rate of temperature increases over a broad range of temperatures. The DC conductivity of all the samples is obtained by measuring current flowing through a piece 


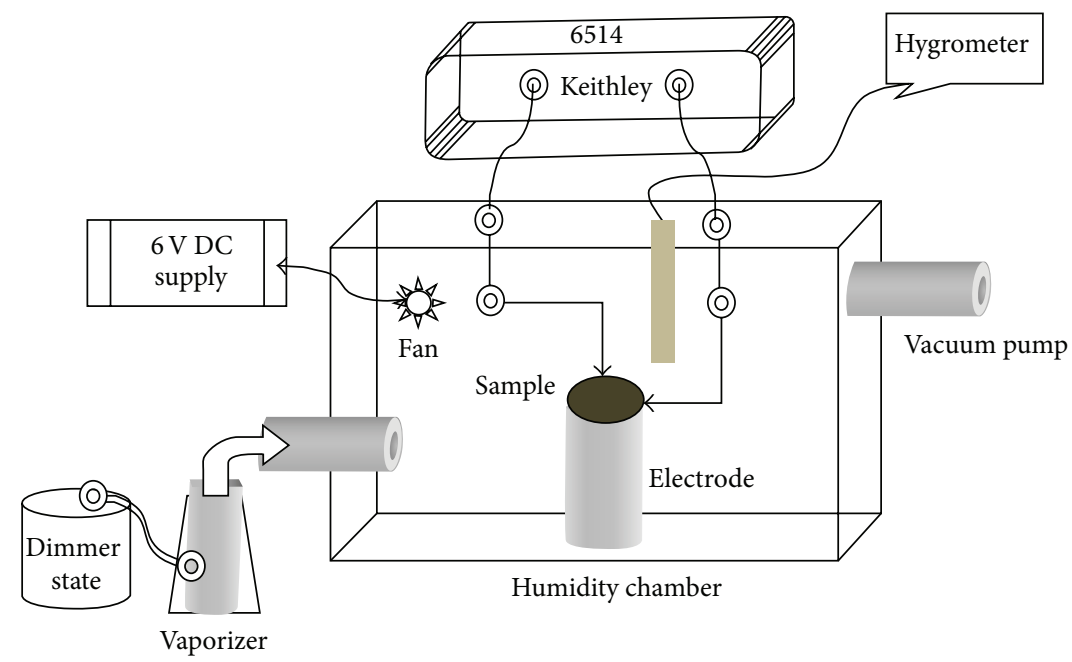

FIGURE 1: Showing the schematic diagram of humidity sensor setup.

of the material and using the sample dimensions $\sigma$ can be calculated using

$$
\sigma=\left(\frac{d}{A V}\right) I \mathrm{~S} \mathrm{~cm}^{-1}
$$

where $d(\mathrm{~cm})$ is the sample thickness. $A$ is its area $\left(\mathrm{cm}^{2}\right), V$ is the potential difference across the material, and $I$ is the current flowing through the material.

3.2. Fabrication of Humidity Sensor Set Up. Humidity sensor chamber is made up of side glass plates of size $250 \mathrm{~mm} \times$ $250 \mathrm{~mm}$ in dimension and $5 \mathrm{~mm}$ thickness provided with top and bottom glass plates. The chamber is made airtight by rubber beading $[13,24]$. The sample holder is then fixed at the central part of the chamber and dc fan at the left corner in order to distribute the humidity uniformly throughout the chamber. The vapors are allowed to pass through the bottom of the left side inlet which is controlled by using dimmer stat. The percentage of humidity in the chamber is monitored by using hygrometer and changes in the resistance of the samples are measured by Keithley meter. Thermal filament is inserted in right side of the bottom connected to the temperature indicator. The right upper side is connected to vacuum pump to dehumidify the chamber for the next cycle/sample. The block diagram of the sensor setup is shown in Figure 1.

\section{Results and Discussion}

4.1. Characterization. Figures 2(a) and 2(b) show the Xray diffraction pattern of (a) $\mathrm{Pr}_{2} \mathrm{O}_{3}$ and (b) PANI- $\mathrm{Pr}_{2} \mathrm{O}_{3}$ composite (30 wt $\%$ of $\operatorname{Pr}_{2} \mathrm{O}_{3}$ in PANI). It is seen from the figure that the cubic peak of praseodymium oxide indicates the crystalline nature of the composite. By comparing the XRD pattern of composite with that of $\mathrm{Pr}_{2} \mathrm{O}_{3}$ (JCPDS No. 47-1111) the prominent peaks corresponding to $2 \theta=26.75^{\circ}$, $56.09^{\circ}$ are due to (100) and (100) planes of $\operatorname{Pr}_{2} \mathrm{O}_{3}$. By comparing the XRD patterns of the composite and $\operatorname{Pr}_{2} \mathrm{O}_{3}$, it is confirmed that $\operatorname{Pr}_{2} \mathrm{O}_{3}$ has retained its structure even though it is dispersed in PANI during polymerization reaction [25].

Figure 3(a,b, and c) shows FTIR spectra of the pure PANI, $\mathrm{Pr}_{2} \mathrm{O}_{3}$, and PANI- $\mathrm{Pr}_{2} \mathrm{O}_{3}$ composite. Figure 3(a) represents the FTIR spectra of pure PANI. The strong peaks in wave number are in the range $1000-1600 \mathrm{~cm}^{-1}$, attributed to the vibrational bands. The band at $504 \mathrm{~cm}^{-1}$ is due to $\mathrm{CH}$ out of plane bending vibration. The bands at 1561 and $1450 \mathrm{~cm}^{-1}$ are attributed to $\mathrm{C}=\mathrm{N}$ and $\mathrm{C}=\mathrm{C}$ stretching mode of vibration for the quinoid and benzenoid units of polyaniline. The bands at 1301 and $1421 \mathrm{~cm}^{-1}$ correspond to the characteristic of pure polyaniline $[26,27]$. The FTIR spectra of the composite (Figure 3(c)) exhibit the characteristic peaks similar to that of pure PANI with a small shift in the characteristic peaks corresponding to that of the pure PANI. The characteristic peaks of $\mathrm{Pr}_{2} \mathrm{O}_{3}$ completely disappear in the IR spectra of composite which reveals the strong interaction between PANI backbone and $\mathrm{Pr}_{2} \mathrm{O}_{3}$ particles during the formation of composite.

Figures 4(a), 4(b), and 4(c) show the surface morphology of pure PANI, $\mathrm{Pr}_{2} \mathrm{O}_{3}$, and PANI- $\mathrm{Pr}_{2} \mathrm{O}_{3}$ composite (50 wt\%) recorded using SEM. Figure 4(a) shows the SEM micrograph of pure PANI with smooth surface having micro pores. The SEM image of $\mathrm{Pr}_{2} \mathrm{O}_{3}$ in Figure 3(b) shows an agglomerated particle structure having uniform distribution. Figure 4(c) shows the SEM micrograph of PANI- $\mathrm{Pr}_{2} \mathrm{O}_{3}$ (30 wt $\%$ ) composite. From the micrograph of composite, one can clearly observe the uniform distribution of $\mathrm{Pr}_{2} \mathrm{O}_{3}$ particles in PANI matrix with the formation of more capillary pores and a conducting network. These capillary pores facilitate the absorption of more water molecules leading to enhanced humidity sensitivity in the composite compared to pure PANI.

4.2. D.C. Conductivity. Figure 5 shows the variation of DC conductivity as a function of temperature for polyaniline and polyaniline- $\mathrm{Pr}_{2} \mathrm{O}_{3}$ composites for different weight percentages. It is observed that the conductivity of polymer composites increases with increase in temperature as well 


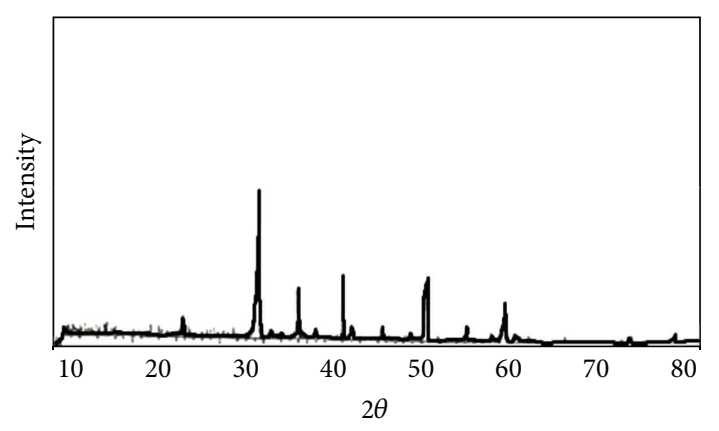

(a)

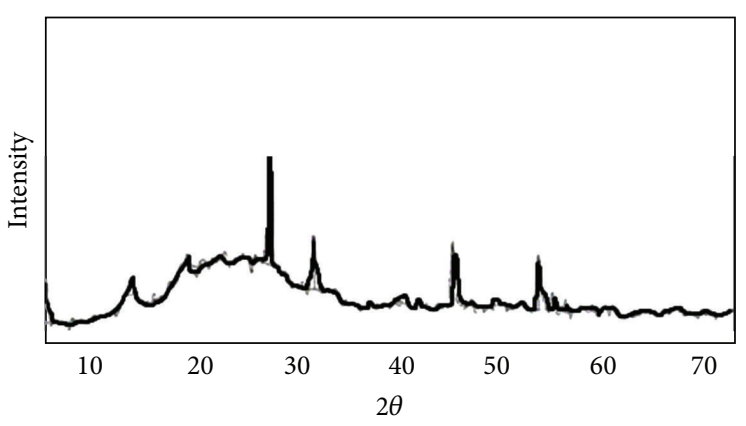

(b)

FIgURE 2: Showing the XRD spectra of (a) $\mathrm{Pr}_{2} \mathrm{O}_{3}$ and (b) PANI- $\mathrm{Pr}_{2} \mathrm{O}_{3}$ composites (30 wt $\%$ ).

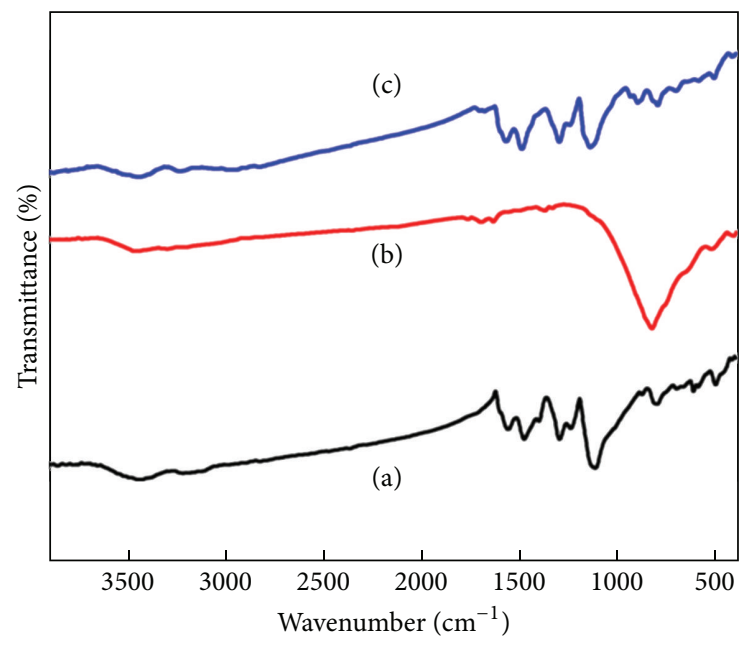

(c)-PP 3

(b) $-\mathrm{Pr}_{2} \mathrm{O}_{3}$

(a)-PANI

FIgURE 3: Showing the FTIR spectra of (a) PANI, (b) $\mathrm{Pr}_{2} \mathrm{O}_{3}$, and (c) PANI- $\mathrm{Pr}_{2} \mathrm{O}_{3}$ composites (30 wt $\%$ ).

as increase in dopant percentages. The three-step conductivity is observed in the range of $30^{\circ} \mathrm{C}$ to $180^{\circ} \mathrm{C}$. In first step, the temperature from $30^{\circ} \mathrm{C}$ to $60^{\circ} \mathrm{C}$ the conductivity of the pure polyaniline and it remains constant because insufficient activation energy fails in hopping of polaron from one island to other [28]. In second step from $60^{\circ} \mathrm{C}$ to $130^{\circ} \mathrm{C}$, the conductivity increases gradually due to the hopping of polarons and bipolarons in short range order. Further, in third step from $130^{\circ} \mathrm{C}$ to $180^{\circ} \mathrm{C}$ the conductivity suddenly increases due to hopping of charge carriers in long range order. The three-step conductivity is characteristic property of amorphous semiconducting materials explained by Mott theory. Among all composites 30 weight percentage polyaniline- $\mathrm{Pr}_{2} \mathrm{O}_{3}$ Composite shows high conductivity due to the elongation of polymer chain in framed network favorable of hopping polarons in long range orders. The conductivity of other composites like 10,20,40, and 50 followed $30 \mathrm{wt} \%$ and its conductivity is lower due to the partial blocking of polarons at the interface of two composite islands. However the expansion of composites is confirmed from thermal coefficient plot as shown in Figure 6. It is observed that the composite shows negative thermal coefficient (NTC) behavior as a function of temperature. The NTC value decreases with increase in metal oxide percentage in polymer matrix due to decrease in the distance between two grains [29]. It is also known that the thermal expansion will cause significant stress in the matrix as the filler does not allow expansion or contraction, which increases the electron density at the junction, causing the blocking of charge carriers [20,30].

4.3. Humidity Sensing Properties. Figure 7 shows the change in resistance as a function of humidity from 15\% RH to $95 \% \mathrm{RH}$ at room temperature. It is found that the change in resistance increases with increase in percentage of relative humidity $(\% \mathrm{RH})$ due to the uncurling of polymer chain giving more favorable path for conduction of charge carriers. The proposed mechanism for change in resistance of these polymer composites is adsorption and desorption of humidity. In lower humidity level the change in the electrical resistance is slow due to the lower adsorption limit (LAL), where uncurling process of the polymer is very slow leading to large distance of hopping sites. At higher humidity, composites absorb more vapor from a long conduction path as shown in Figure 8. Hence change in resistance decreases suddenly [31]. It is important to note that the pure polymer shows large change in resistance but above $30 \% \mathrm{RH}$ the polymer becomes unstable and starts degrading as results change in resistance almost constant. Among all composites, $30 \mathrm{wt} \%$ of polyaniline- $\mathrm{Pr}_{2} \mathrm{O}_{3}$ composite shows linear decrease of change in resistance throughout the range indicating that these composites are more stable and help in holding the vapor molecules. Another possibility is when composites are exposed to humidity, in the first stage of the interaction a few water vapour molecules are chemically adsorbed (chemisorption) at the neck of the crystalline grains on activated sites of the surface, which is accompanied with a dissociative mechanism of vapour molecules to form hydroxyl groups (two hydroxyl ions per water molecule). As an interaction between the surface ions of the grain necks and the adsorbed water, the hydroxyl group of each water molecule is adsorbed on metal cations which are present in the grains' surfaces and possess high charge carrier density 


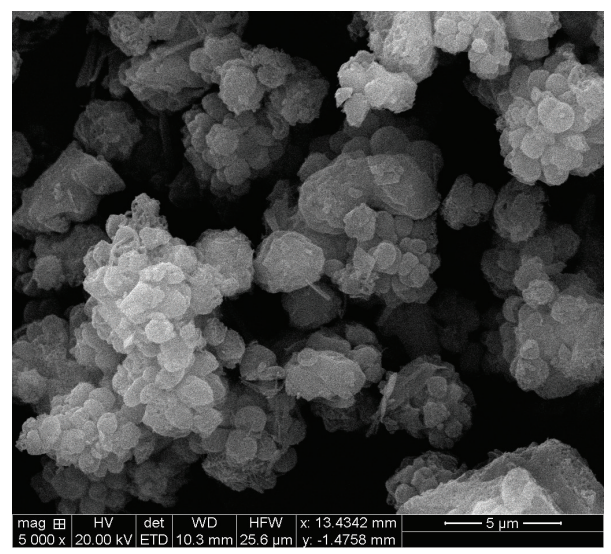

(a)

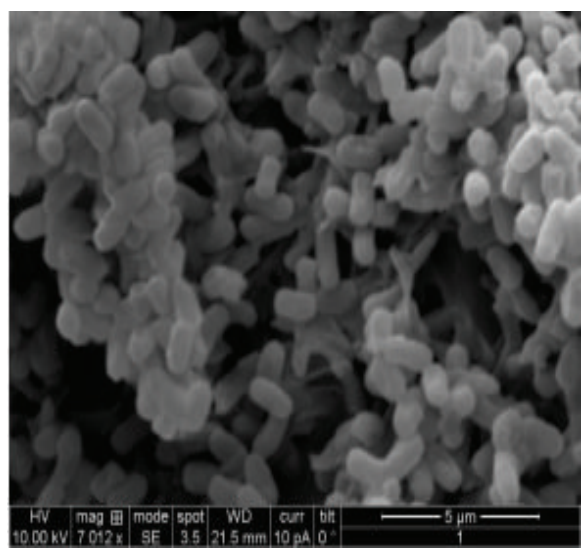

(b)

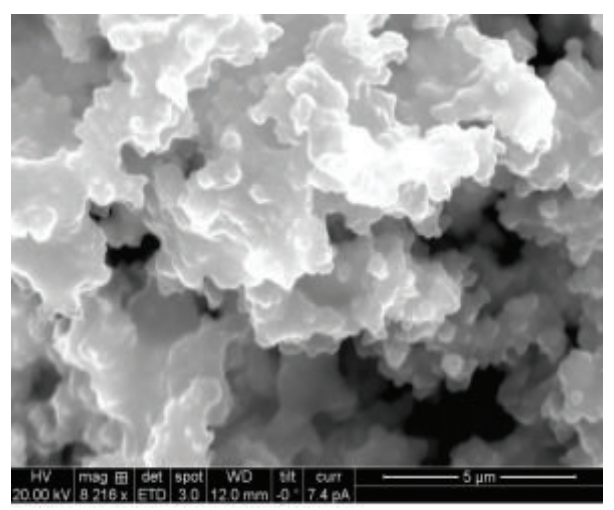

(c)

FIgure 4: Showing the SEM image of (a) PANI, (b) $\mathrm{Pr}_{2} \mathrm{O}_{3}$, and (c) PANI- $\mathrm{Pr}_{2} \mathrm{O}_{3}$ composites (30 wt\%).

and strong electrostatic fields, thus providing mobile protons. The protons migrate from site to site on the surface and react with the neighbour surface $\mathrm{O}^{2-}$ groups (oxygen) to form a second hydroxyl $\left(\mathrm{OH}^{-}\right)$group. The chemisorbed layer is the first formed layer so once it has formed on the surface it will not change further by exposure to humidity. As a second stage, after chemical completion of the first layer, subsequent water vapour layers are physically adsorbed (physisorption) on the first formed hydroxyl layer and stack to form the physisorbed hydroxyl multilayer. After forming the first physisorbed layer, another water molecule adsorbs via double hydrogen bonding to two neighbouring hydroxyl groups. As water vapour continues to increase in the surface, an extra layer forms on the first physisorbed layer; therefore the physisorption changes from monolayer to multilayer. These layers are less ordered than the first physisorbed layer, and water vapour molecules in these layers may be only singly bonded to local hydrogens.

Finally, by forming more layers, a large amount of water molecules is physisorbed on the necks and flat surfaces, hence singly bonded water vapor molecules become mobile and able to form continuous dipoles and electrolyte layers between the electrodes, resulting in an increased dielectric constant and bulk conductivity. Therefore, the slight variations of conductivity with humidity adsorption can be due to a water protonation and polarons conduction mechanism on the composite surface.

The sensitivity of detecting the humidity is defined by the percentage change in resistance per $10 \%$ change in the value of relative humidity [32]. The variations in the resistivity as a function of relative humidity $(\mathrm{RH})$ value for $\mathrm{PANI} / \mathrm{Pr}_{2} \mathrm{O}_{3}$ composite in the form of pellets for five different weight percentages $(10,20,30,40$, and $50 \mathrm{wt} \%)$. The percentage of sensitivity for detection of humidity is defined as

$$
S=\frac{\mathrm{RH}_{2}-\mathrm{RH}_{1}}{\mathrm{RH}_{1}} \times 100,
$$

where $\mathrm{RH}_{2}$ is the resistivity of the sample for humidity at level 2 (highest $\mathrm{RH}$ level) and $\mathrm{RH}_{1}$ is the resistivity of the sample for humidity at level 1 (lowest RH level). Figure 9 shows the sensitivity of polyaniline and polyaniline- $\mathrm{Pr}_{2} \mathrm{O}_{3}$ composites as a function of relative humidity for different weight percentages. The sensitivity is measured from RH $20 \%$ to $\mathrm{RH} 95 \%$. It is found that the sensitivity increases with increase in relative humidity. Among all composites $30 \mathrm{wt} \%$ shows high sensitivity and more stability in long range of $\mathrm{RH} \%$ followed by other composites which shows almost similar behavior $[33,34]$. These composites above $30 \mathrm{wt} \%$ the absorption is good but as the polymer percentages is more than metal oxide fails to hold the water vapor for longer time 


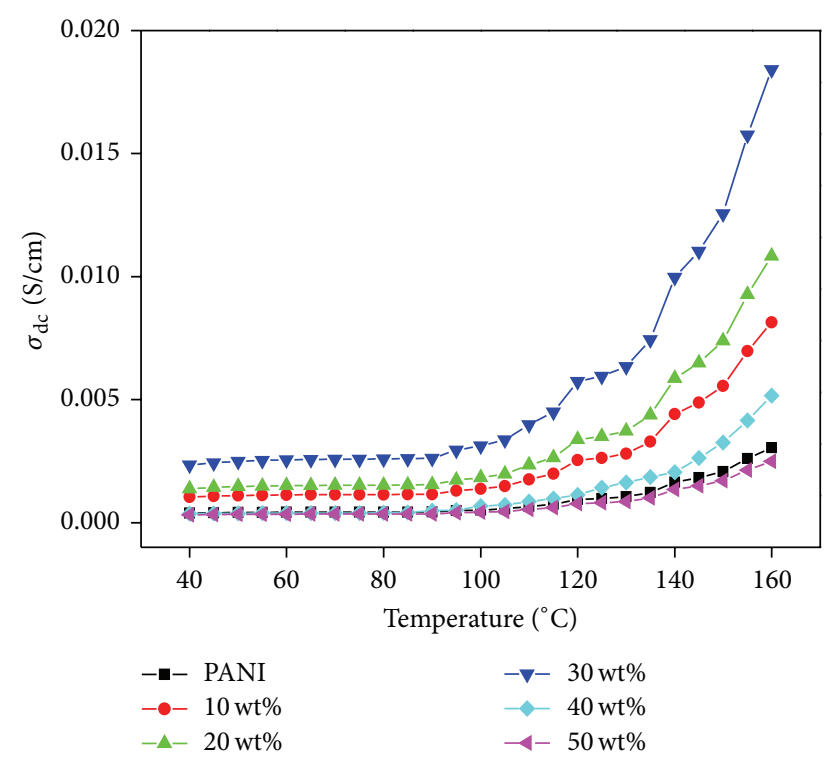

Figure 5: Showing the DC conductivity of PANI and PANI- $\mathrm{Pr}_{2} \mathrm{O}_{3}$ composites for various $w t \%$.

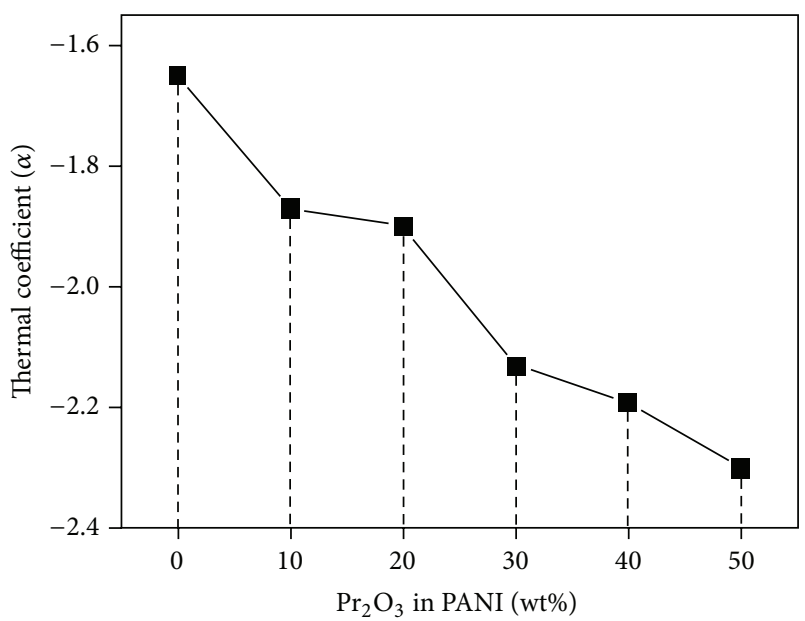

FIgure 6: Thermal coefficient of PANI and PANI- $\mathrm{Pr}_{2} \mathrm{O}_{3}$ composites for various wt\%.

as a results after some period of exposing with humidity starts saturating the surface. Hence sensitivity decreases. In case of pure polyaniline sensitivity even more than composites but the stability of the polyaniline loses above $50 \% \mathrm{RH}$ and starts degradation of polymer.

The vapor is removed from the humidity chamber by using vacuum pump but we cannot remove it completely from the samples. Therefore once the humidity of chamber attends $40 \% \mathrm{RH}$, heating should be switched on till the electrical resistance comes to base level in order to start next cycle. Figure 10 shows the response and recovery curves corresponding to water adsorption and desorption process for $10,20,30,40$, and $50 \mathrm{wt} \%$ of $\mathrm{PANI} / \mathrm{Pr}_{2} \mathrm{O}_{3}$ composites. The average response time of $\mathrm{PANI} / \mathrm{Pr}_{2} \mathrm{O}_{3}$ composite is 377

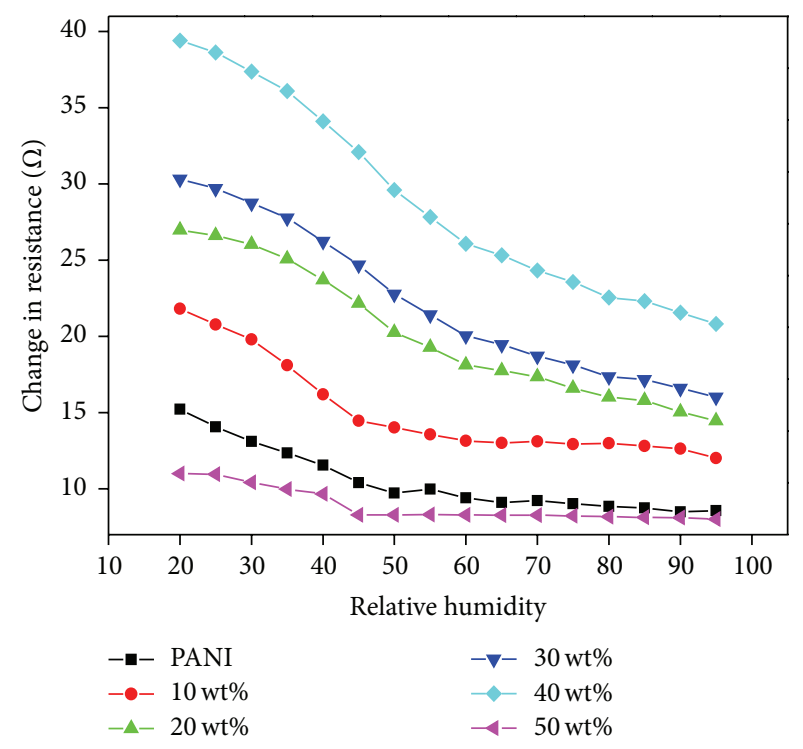

FIGURE 7: Change in resistance as function of relative humidity of PANI and PANI- $\mathrm{Pr}_{2} \mathrm{O}_{3}$ composites.
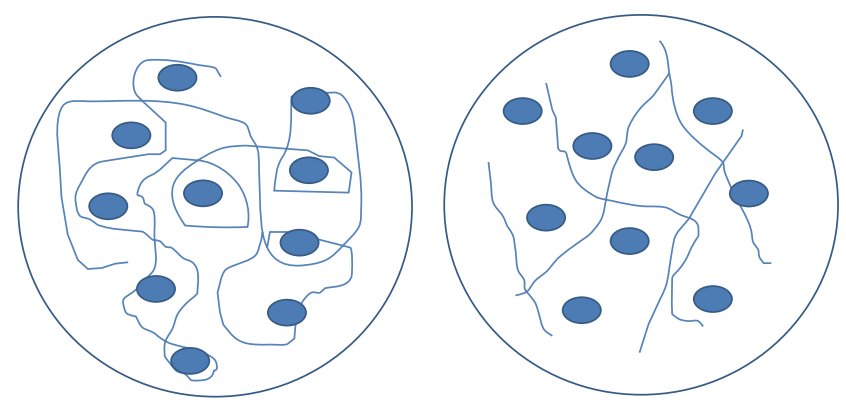

FIGURE 8: Schematic illustration of humidity absorption in polyaniline composites.

seconds and the recovery time is 453 seconds as the humidity changes from $15 \%$ to $95 \%$ RH. Among all composites $30 \mathrm{wt} \%$ shows first response to humidity and less recovery time. Therefore these polymer composites can be used in food industries, domestic storage chamber, packing industries, solar cell, battery, and so forth.

\section{Conclusions}

In this paper, we have reported humidity sensing properties of polyaniline- $\mathrm{Pr}_{2} \mathrm{O}_{3}$ composites prepared by in situ polymerization. The SEM micrograph of the composite shows the encapsulation of $\mathrm{Pr}_{2} \mathrm{O}_{3}$ in PANI matrix and reveals the presence of capillary pores which facilitate the sensing behavior. From the XRD spectrum of PANI- $\mathrm{Pr}_{2} \mathrm{O}_{3}$ composite, it is evident that with the $\operatorname{Pr}_{2} \mathrm{O}_{3}$ content in PANI, the peak intensity corresponding to both PANI and $\operatorname{Pr}_{2} \mathrm{O}_{3}$ is modified which confirms coexistence of PANI and $\operatorname{Pr}_{2} \mathrm{O}_{3}$ phase in the composite. The FTIR spectrum reveals the formation of composite with strong interaction between PANI chains and $\operatorname{Pr}_{2} \mathrm{O}_{3}$ particles. The humidity sensing mechanism is 


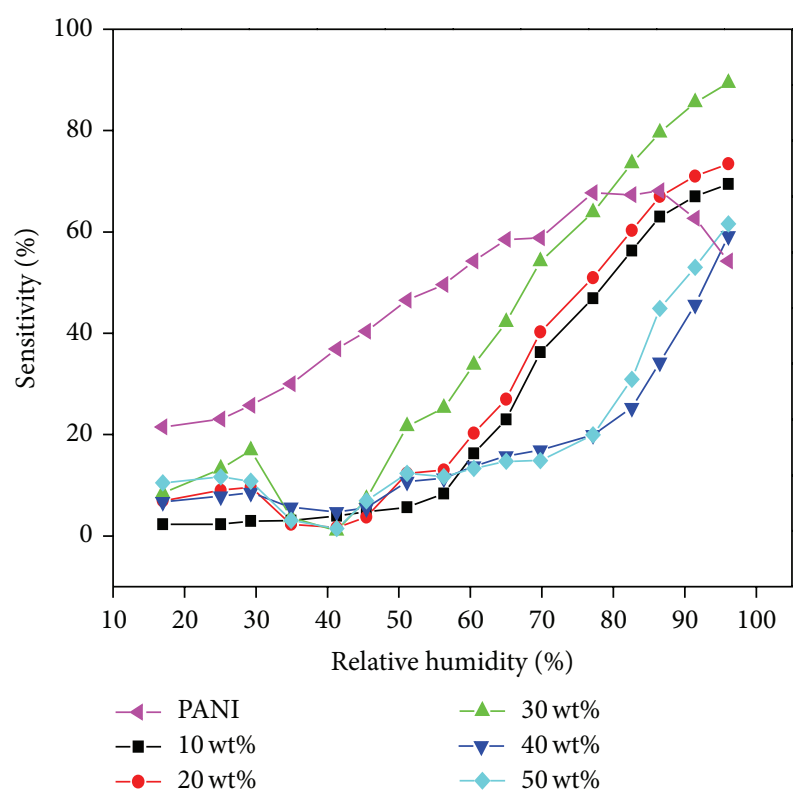

FIGURE 9: Variation of sensitivity against relative humidity for PANI and its composites.

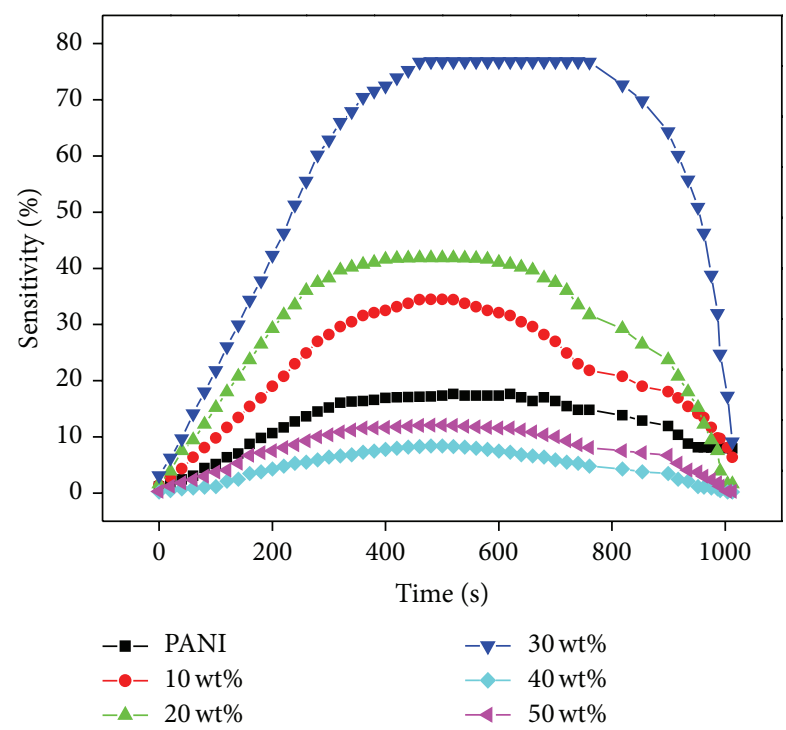

FIGURE 10: Showing the response and recovery time against time of PANI and PANI- $\mathrm{Pr}_{2} \mathrm{O}_{3}$ composites.

investigated using homemade two-probe method. The studies on temperature dependent conductivity of the composite at various humidity levels have shown the increase in sensitivity for the composite with increase in \% RH. Among all composites, 30 wt $\%$ of PANI- $\operatorname{Pr}_{2} \mathrm{O}_{3}$ composite shows high sensitivity and exhibits the maximum humidity sensing response and shorter response-recovery time. Hence our studies suggest that PANI- $\mathrm{Pr}_{2} \mathrm{O}_{3}$ composite can be a promising material for high performance humidity sensing applications.

\section{Conflict of Interests}

The authors declare that there is no conflict of interests regarding the publication of this paper.

\section{References}

[1] S. Park, J. Kang, J. Park, and S. Mun, "One-bodied humidity and temperature sensor having advanced linearity at low and high relative humidity range," Sensors and Actuators $B$, vol. 76, no. 1-3, pp. 322-326, 2001.

[2] A. T. Ramaprasad and V. Rao, "Chitin-polyaniline blend as humidity sensor," Sensors and Actuators B: Chemical, vol. 148, no. 1, pp. 117-125, 2010.

[3] C. Y. Lee and G. B. Lee, "Humidity sensors: a review," Sensor Letters, vol. 3, no. 1, pp. 1-15, 2005.

[4] P.-G. Su and Y.-P. Chang, "Low-humidity sensor based on a quartz-crystal microbalance coated with polypyrrole/Ag/ $/ \mathrm{TiO}_{2}$ nanoparticles composite thin films," Sensors and Actuators B: Chemical, vol. 129, no. 2, pp. 915-920, 2008.

[5] A. Batool, F. Kanwal, M. Imran, T. Jamil, and S. A. Siddiqi, "Synthesis of polypyrrole/zinc oxide composites and study of their structural, thermal and electrical properties," Synthetic Metals, vol. 161, no. 23-24, pp. 2753-2758, 2012.

[6] M. Ferenets and A. Harlin, "Chemical in situ polymerization of polypyrrole on poly(methyl metacrylate) substrate," Thin Solid Films, vol. 515, no. 13, pp. 5324-5328, 2007.

[7] A. Roy, A. Parveen, R. Deshpande, R. Bhat, and A. Koppalkar, "Microscopic and dielectric studies of $\mathrm{ZnO}$ nanoparticles loaded in ortho-chloropolyaniline nanocomposites," Journal of Nanoparticle Research, vol. 15, no. 1, article 1337, 2013.

[8] S. K. Mahadeva, S. Yun, and J. Kim, "Flexible humidity and temperature sensor based on cellulose-polypyrrole nanocomposite," Sensors and Actuators A: Physical, vol. 165, no. 2, pp. 194-199, 2011.

[9] W. Zhang, R. Wang, Q. Zang, and J. Li, "Humidity sensitive properties of K-doped mesoporous silica SBA-15," Journal of Physics and Chemistry of Solids, vol. 73, pp. 517-522, 2012.

[10] A. Parveen, A. Koppalkar, and A. S. Roy, "Liquefied petroleum gas sensing of polyaniline-titanium dioxide nanocomposites," Sensor Letters, vol. 11, no. 2, pp. 242-248, 2013.

[11] P. G. Su and C. P. Wang, "Flexible humidity sensor based on $\mathrm{TiO}_{2}$ nanoparticles-polypyrrole-poly-[3-(methacrylamino) propyl] trimethyl ammonium chloride composite materials," Sensors and Actuators B: Chemical, vol. 129, no. 2, pp. 538-543, 2008.

[12] R. P. Tandon, M. R. Tripathy, A. K. Arora, and S. Hotchandani, "Gas and humidity response of iron oxide-polypyrrole nanocomposites," Sensors and Actuators B: Chemical, vol. 114, no. 2, pp. 768-773, 2006.

[13] N. Yamazoe and Y. Shimizu, "Humidity sensors: principles and applications," Sensors and Actuators B, vol. 10, no. 3-4, pp. 379398, 1986.

[14] N. Parvatikar, S. Jain, S. Khasim, M. Revansiddappa, S. V. Bhoraskar, and M. V. N. A. Prasad, "Electrical and humidity sensing properties of polyaniline/ $\mathrm{WO}_{3}$ composites," Sensors and Actuators B: Chemical, vol. 114, no. 2, pp. 599-603, 2006.

[15] N. Parvatikar, S. Jain, C. M. Kanamadi, B. K. Chougule, S. V. Bhoraskar, and M. V. N. A. Prasad, "Humidity sensing and electrical properties of polyanilme/cobalt oxide composites," Journal of Applied Polymer Science, vol. 103, no. 2, pp. 653-658, 2007. 
[16] N. Parvatikar, S. Jain, S. V. Bhoraskar, and M. V. N. Ambika Prasad, "Spectroscopic and electrical properties of polyaniline/ $\mathrm{CeO}_{2}$ composites and their application as humidity sensor," Journal of Applied Polymer Science, vol. 102, no. 6, pp. 5533-5537, 2006.

[17] D. Patil, Y.-K. Seo, Y. K. Hwang, J.-S. Chang, and P. Patil, "Humidity sensing properties of poly $(o$-anisidine $) / \mathrm{WO}_{3} \mathrm{com}-$ posites," Sensors and Actuators B, vol. 128, no. 2, pp. 374-382, 2008.

[18] L. Geng, Y. Zhao, X. Huang, S. Wang, S. Zhang, and S. Wu, "Characterization and gas sensitivity study of polyaniline/ $\mathrm{SnO}_{2}$ hybrid material prepared by hydrothermal route," Sensors and Actuators B: Chemical, vol. 120, no. 2, pp. 568-572, 2007.

[19] L. Yuan, J. Wang, S. Y. Chew et al., "Synthesis and characterization of $\mathrm{SnO}_{2}$-polypyrrole composite for lithium-ion battery," Journal of Power Sources, vol. 174, no. 2, pp. 1183-1187, 2007.

[20] P.-G. Su and L.-N. Huang, "Humidity sensors based on $\mathrm{TiO}_{2}$ nanoparticles/polypyrrole composite thin films," Sensors and Actuators B: Chemical, vol. 123, no. 1, pp. 501-507, 2007.

[21] M. L. Singla, S. Awasthi, and A. Srivastava, "Humidity sensing; using polyaniline/ $\mathrm{Mn}_{3} \mathrm{O}_{4}$ composite doped with organic/inorganic acids," Sensors and Actuators B: Chemical, vol. 127, no. 2, pp. 580-585, 2007.

[22] S. J. Varma and S. Jayalakshmi, "On the prospects of polyaniline and polyaniline/MWNT composites for possible pressure sensing applications," Journal of Applied Polymer Science, vol. 117, no. 1, pp. 138-142, 2010.

[23] A. S. Roy, S. G. Hegde, and A. Parveen, "Synthesis, characterization, AC conductivity, and diode properties of polyaniline$\mathrm{CaTiO}_{3}$ composites," Polymers for Advanced Technologies, vol. 25, no. 1, pp. 130-135, 2014.

[24] M. Ghorbani and H. Eisazadeh, "Synthesis and characterization of chemical structure and thermal stability of nanometer size polyaniline and polypyrrole coated on rice husk," Synthetic Metals, vol. 162, no. 5-6, pp. 527-530, 2012.

[25] A. S. Roy, A. Parveen, A. Prasad, and K. R. Anilkumar, "Influence of $\mathrm{Ta}_{2} \mathrm{O}_{5}$ on polyaniline surface for liquid petroleum gas sensing applications," Sensor Review, vol. 32, no. 2, pp. 163$169,2012$.

[26] S. Chaudhari, P. P. Patil, A. B. Mandale, K. R. Patil, and S. R. Sainkar, "Use of poly(o-toluidine) $/ \mathrm{ZrO}_{2}$ nanocomposite coatings for the corrosion protection of mild steel," Journal of Applied Polymer Science, vol. 106, no. 1, pp. 220-229, 2007.

[27] A. S Roy, S. Gupta, P. C. Ramamurthy, and G. Madras, "Fabrication of poly(vinylidene chloride-co-vinyl chloride)/ $/ \mathrm{TiO}_{2}$ nanocomposite films and their dielectric properties," Science of Advanced Materials, vol. 6, pp. 946-953, 2014.

[28] A. Parveen, A. R. Koppalkar, and A. S. Roy, "Surface modified $\mathrm{CaTiO}_{3}$ loaded in polyaniline by sodium dodecyl benzene sulphonic acid for humidity sensor," IEEE Sensors Journal, vol. 12, no. 9, pp. 2817-2823, 2012.

[29] C.-Y. Lee and G.-B. Lee, "Humidity sensors: a review," Sensor Letters, vol. 3, no. 1, pp. 1-15, 2005.

[30] T. MacHappa and M. V. N. A. Prasad, "Low frequency AC conduction in polyaniline/Zinc tungstate $\left(\mathrm{PANI} / \mathrm{ZnWO}_{4}\right) \mathrm{com}$ posites," Ferroelectrics, vol. 392, no. 1, pp. 71-80, 2009.

[31] H. M. Kim, C. Y. Lee, and J. Joo, "AC dielectric relaxation of lightly hydrochloric-acid( $\mathrm{HCl})$-doped polyanilines," The Korean Physical Society, vol. 36, pp. 71-376, 2000.

[32] R. Patil, A. S. Roy, K. R. Anilkumar, and S. Ekhelikar, "Studies on Fourier transform infrared spectroscopy, scanning electron microscope, and direct current conductivity of polyaniline doped zinc ferrite," Journal of Applied Polymer Science, vol. 121, no. 1, pp. 262-266, 2011.

[33] A. S. Roy, K. R. Anilkumar, and M. V. N. A. Prasad, "Core-shell method of synthesis, characterizations, and ac conductivity studies of polyaniline/n- $-\mathrm{TiO}_{2}$ composites," Journal of Applied Polymer Science, vol. 121, no. 2, pp. 675-680, 2011.

[34] S. Agarwal and G. L. Sharma, "Humidity sensing properties of $(\mathrm{Ba}, \mathrm{Sr}) \mathrm{TiO}_{3}$ thin films grown by hydrothermalelectrochemical method," Sensors and Actuators B: Chemical, vol. 85, no. 3, pp. 205-211, 2002. 

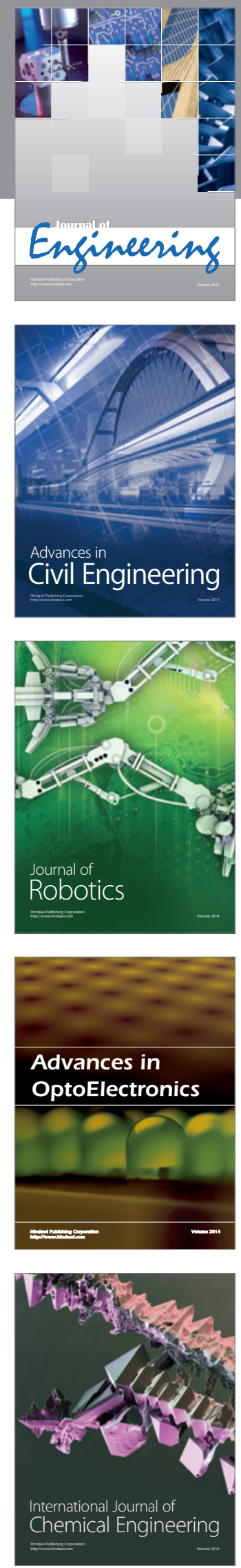

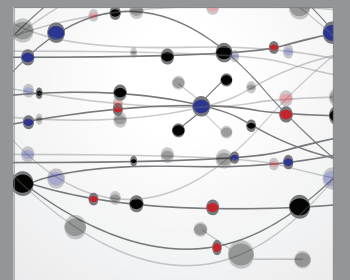

The Scientific World Journal
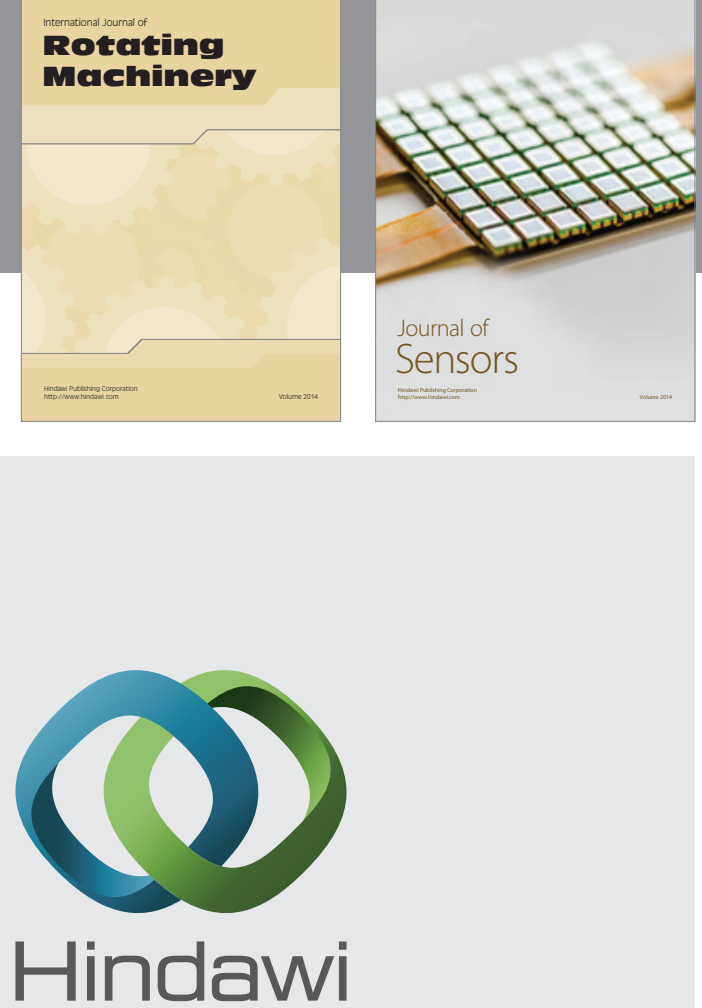

Submit your manuscripts at http://www.hindawi.com
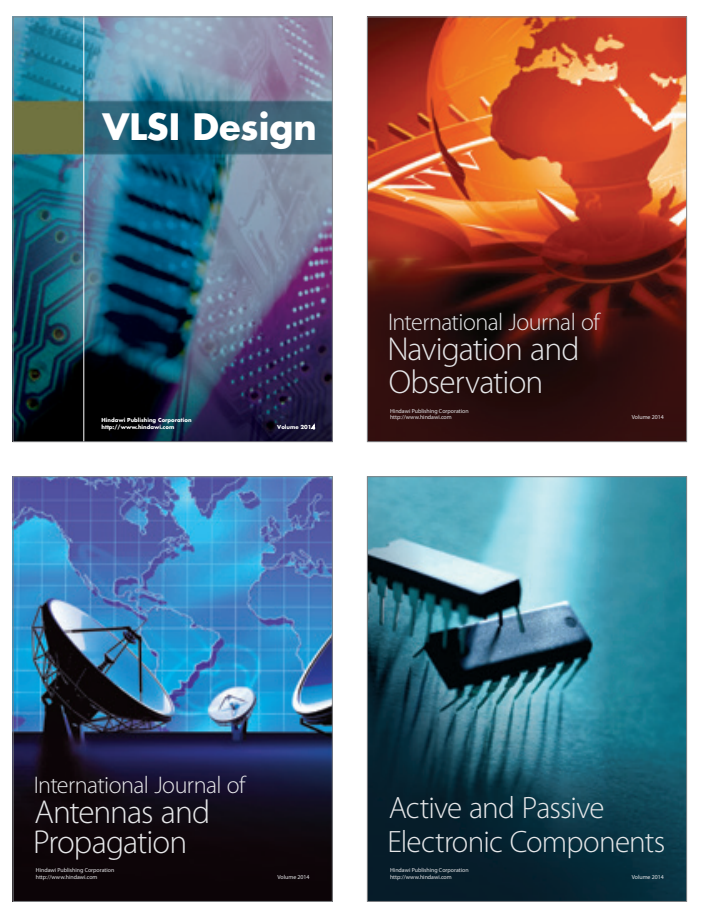
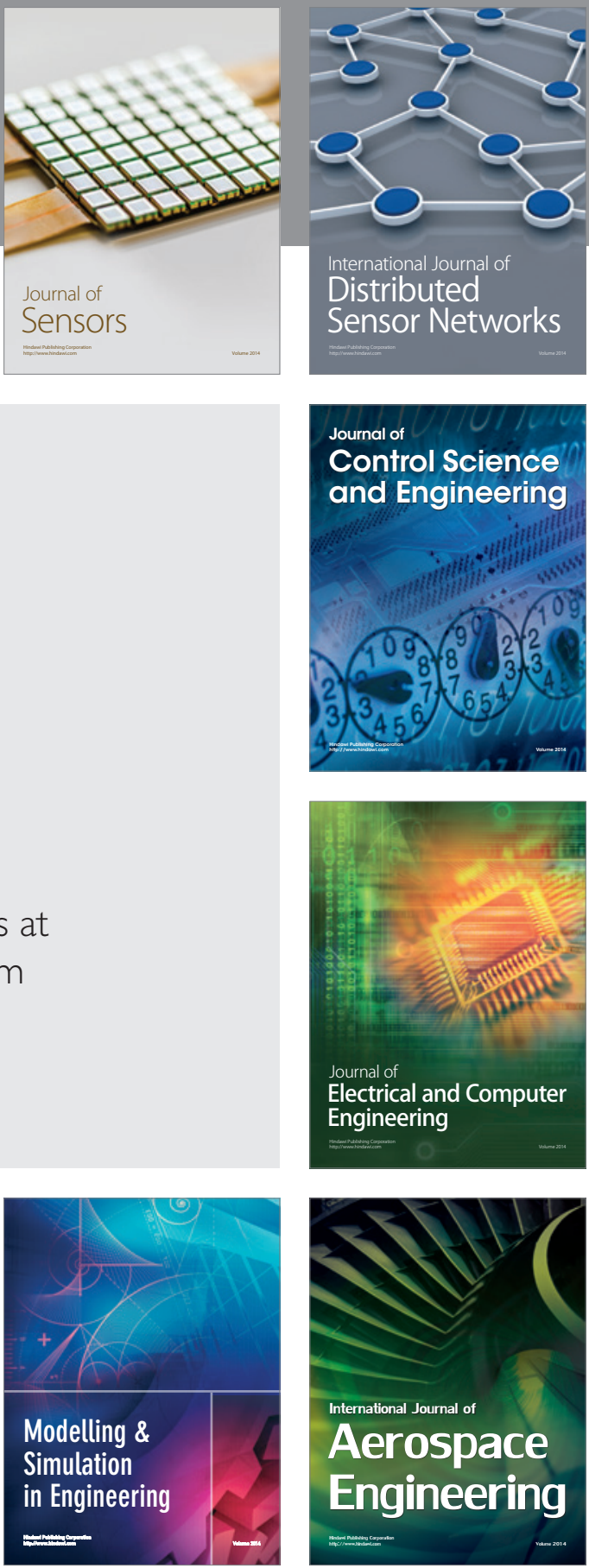

Journal of

Control Science

and Engineering
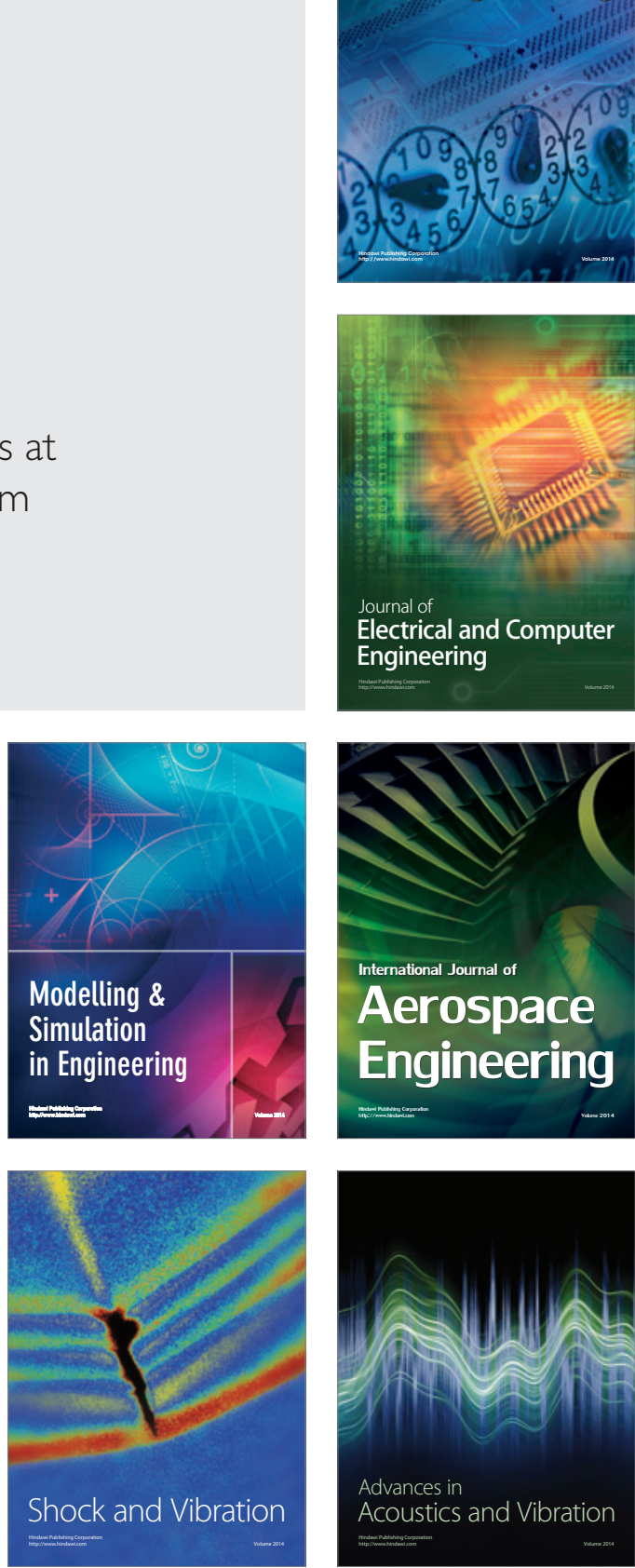